\title{
Multiple beam envelope equations for electron injectors using a bunch segmentation model
}

\author{
A. Mizuno, ${ }^{*}$ H. Dewa, T. Taniuchi, H. Tomizawa, and H. Hanaki \\ JASRI/SPring-8, 1-1-1, Koto, Sayo, Hyogo, 679-5198, Japan \\ E. Hotta \\ Department of Energy Sciences, Tokyo Institute of Technology, Nagatsuta-cho, 4259, J2-35, \\ Midori-ku, Yokohama, Kanagawa, 226-8502, Japan \\ (Received 4 April 2012; published 7 June 2012)
}

\begin{abstract}
A new semianalytical method of investigating the beam dynamics for electron injectors was developed. In this method, a short bunched electron beam is assumed to be an ensemble of several segmentation pieces in both the longitudinal and the transverse directions. The trajectory of each electron in the segmentation pieces is solved by the beam envelope equations while taking into account the space charge fields produced by all the pieces, the electromagnetic fields of an rf cavity, and the image charge fields at a cathode surface. The shape of the entire bunch is consequently calculated, and thus the emittances can be obtained from weighted mean values of the solutions for the obtained electron trajectories. The advantage of this method is its unique assumption for the beam parameters. We assume that each segmentation slice is not warped in the calculations. Although if the beam energy is low and the charge density is large, this condition is not satisfied, in practice, this condition is usually satisfied. We have performed beam dynamics calculations to obtain traces in free space and in the BNL-type rf gun cavity by comparing the analytical solutions with those obtained by simulation. In most cases, the emittances obtained by the simulation become closer to those obtained analytically with increasing the number of particles used in the simulation. Therefore, the analytically obtained emittances are expected to coincide with converged values obtained by the simulation. The applicable range of the analytical method for the BNL-type rf gun cavity is under $0.5 \mathrm{nC}$ per bunch. This range is often used in recently built $\mathrm{x}$-ray free electron laser facilities.
\end{abstract}

DOI: 10.1103/PhysRevSTAB.15.064201

\section{INTRODUCTION}

The emittance calculation technique plays an important role in the design of electron injectors, particularly, very low emittance electron sources such as $\mathrm{x}$-ray free electron lasers (XFELs) [1-3]. There have been many reports on envelope equations and analytical solutions for beam dynamics [4-8], and the time evolution of emittance can be calculated even in rf structures and magnetic fields.

For example, in Ref. [6], longitudinal slices of a bunch are assumed. The envelope equations used to calculate the rms beam size of the bunch were analytically derived from an ensemble of each slice behavior. The space charge effects including the correlations among each slice were analytically taken into account by considering the geometrical factor of the bunch. The evolution of the transverse rms emittance can be derived from the solutions of the rms

\footnotetext{
*Also at Department of Energy Sciences, Tokyo Institute of Technology, Kanagawa, Japan. mizuno@ spring8.or.jp

Published by the American Physical Society under the terms of the Creative Commons Attribution 3.0 License. Further distribution of this work must maintain attribution to the author( $s)$ and the published article's title, journal citation, and DOI.
}

PACS numbers: 29.25.Bx, 29.27.- a, 41.75.-i, 41.85.Ja

envelopes. The effects of rf fields can also be analytically taken into account.

However, it is difficult to accurately calculate practical bunch shapes by the previous analytical methods, and the detailed emittance behavior calculated by these methods does not agree with that of practical beams. Therefore, it has been common to use simulation codes to perform accurate calculations.

Some particle-tracking codes [9-13] can be used to perform three-dimensional simulations, and can even calculate the complex bunch shapes of practical beams. However, in these methods, the calculated emittances are often dependent on the number of particles. This is mainly caused by the random noise in the particle position distribution or in treatments for calculating the space charge effects among closely located particles. To enhance the accuracy of the calculated emittance, many particles are required, which increases the computation time. In addition, depending on the initial parameters of the simulation, emittances may not converge to the true values.

In contrast, in this paper we overcome this problem by combining an analytical method with a simulation method to accurately calculate the bunch shape and remove the dependence on the number of particles. 
We introduce a bunch segmentation model that considers segmentation pieces in both the longitudinal and the transverse directions. Electrons are located at each segmentation piece. The trajectory of each electron is calculated by multiple beam envelope equations, which are simultaneous ordinary differential equations, assuming interaction among the longitudinal segments. Here, these envelope equations describe not for the rms beam size but for each electron trajectory, and the bunch shape can be obtained from these trajectories. The transverse projected emittance of the bunch can be obtained from the weighted mean of the electron trajectories.

To consider dynamics in external fields such as rf fields, we include actual numerical field data in the envelope equations instead of using analytical field calculations to obtain realistic solutions. Image charge effects at the cathode surface, which is assumed to be a perfect conductor, are taken into account in the equations by calculating the fields from the image charge of each longitudinal segment, making it possible to calculate the emission from the cathode.

In Sec. II, beam envelope equations, which can be applied for a pulsed beam regardless of its energy or longitudinal length, are derived. These equations are a set of transverse and longitudinal simultaneous differential equations in the laboratory frame.

In the analysis in Sec. III, we divide a pulsed beam into several longitudinal slices, and derive multiple beam envelope equations, which can be used to calculate the bunch shape and its projected emittance. In this section, we introduce a transverse space charge field correction scheme used to ensure accurate calculations.

In Sec. IV, we show the accuracy of the proposed method by comparing results for emittances obtained by the multiple beam envelope equations with those results obtained by a particle-tracking code. We also give the calculated beam dynamics in the BNL-type rf gun cavity and discuss the applicable range of the multiple envelope equations.

\section{BEAM ENVELOPE EQUATIONS}

\section{A. Concept of transverse beam envelope equation}

Although an electron beam has a finite radius of $R$, in practice it is assumed to be a one-dimensional pencil beam as shown in Fig. 1. A transverse beam envelope equation can be obtained by tracing an electron trajectory while

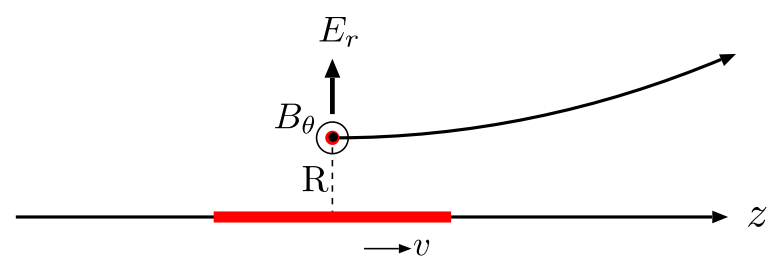

FIG. 1. Model used to derive the transverse beam envelope equation. taking into account the space charge fields produced by the pencil beam located along the $z$ axis. The electron is initially located at a distance of $R$ from the beam axis.

The equation of motion of the electron is

$$
\frac{d\left(\gamma m_{0} \boldsymbol{v}\right)}{d t}=-e(\boldsymbol{v} \times \boldsymbol{B}+\boldsymbol{E}),
$$

where $e$ is the electron charge, $\boldsymbol{v}$ is the electron velocity, $\gamma m_{0} \boldsymbol{v}$ is the electron momentum, and $\boldsymbol{E}$ and $\boldsymbol{B}$ are the electric and magnetic fields, respectively. From Eq. (1),

$$
\frac{d \boldsymbol{v}}{d t}=-\frac{e}{\gamma m_{0}}\left(\boldsymbol{v} \times \boldsymbol{B}+\boldsymbol{E}-\frac{(\boldsymbol{v} \cdot \boldsymbol{E})}{c^{2}} \boldsymbol{v}\right),
$$

where $c$ is the velocity of light in vacuum. Here, we consider only $E_{r \cdot s c}$ and $B_{\theta \cdot s c}$ as space charge fields and that $B_{\theta \cdot s c}=\frac{\beta}{c} E_{r \cdot s c}$. Ignoring $E_{\theta}, B_{z}$, and $\left(\frac{v_{r}}{c}\right)^{2}$, the $r$ component of Eq. (2) can be expressed as

$\frac{d^{2} R}{d t^{2}}=-\frac{e}{\gamma^{3} m_{0}} E_{r \cdot s c}-\frac{e}{\gamma m_{0}}\left(E_{r}-\beta c B_{\theta}-\frac{\beta}{c} \frac{d R}{d t} E_{z}\right)$,

where $\beta=v_{z} / c$ and $E_{r}, B_{\theta}$, and $E_{z}$ are external fields caused by factors other than the space charge.

For a continuous beam, $B_{\theta \cdot s c}$ can be calculated from Ampère's law,

$$
B_{\theta \cdot s c}=-\frac{\mu_{0} I}{2 \pi R}
$$

where $I$ is the current of the continuous beam. Therefore, Eq. (3) becomes

$\frac{d^{2} R}{d t^{2}}=\frac{e I}{2 \pi \epsilon_{0} m_{0} \beta \gamma^{3} c R}-\frac{e}{\gamma m_{0}}\left(E_{r}-\beta c B_{\theta}-\frac{\beta}{c} \frac{d R}{d t} E_{z}\right)$.

The first term of Eq. (5) is the space charge term and agrees with that of Lawson's envelope equation [4] because it is for a continuous beam.

\section{B. Transverse envelope equation for pulsed beam}

To obtain an envelope equation for a pulsed beam, the electric field from the pulsed beam should be derived.

First, an electric field in the laboratory frame, which is produced by an electron with linear uniform motion and velocity $\boldsymbol{v}$, is generally given as

$$
\boldsymbol{E}(\boldsymbol{R})=\frac{1}{4 \pi \epsilon_{0} \gamma^{2}} \frac{-e \boldsymbol{R}}{\left[|\boldsymbol{R}|^{2}-\frac{|\boldsymbol{v} \times \boldsymbol{R}|^{2}}{c^{2}}\right]^{3 / 2}},
$$

where $\boldsymbol{R}$ is a vector from the present position of the electron. This expression is derived from the LiénardWiechert potentials but all variables are defined at the present time.

For a uniformly charged pulsed pencil beam with a length of $L$, a total charge of $Q$ and an energy of $\gamma m_{0} c^{2}$, as shown in Fig. 2, $d E_{r}$ at point $O\left(z_{0}, R\right)$ produced by the charges located within width $d z$ is calculated from Eq. (6) as follows: 


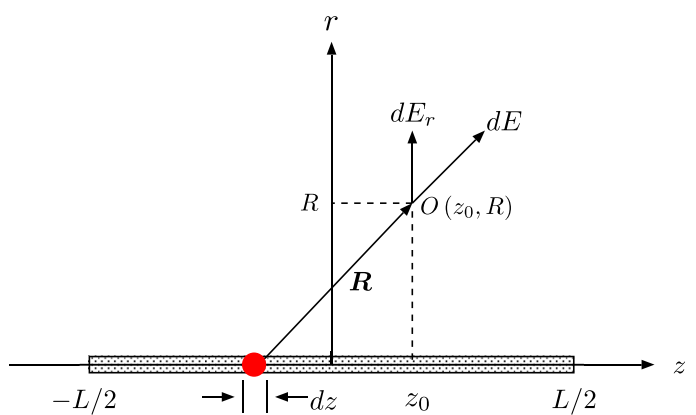

FIG. 2. Calculation model of space charge field generated by a pencil beam.

$$
d E_{r}=-\frac{1}{4 \pi \epsilon_{0} \gamma^{2}} \cdot \frac{Q}{L} d z \cdot \frac{R}{\left[\left(z_{0}-z\right)^{2}+\frac{R^{2}}{\gamma^{2}}\right]^{3 / 2}} .
$$

$E_{r \cdot s c}$ is derived by integrating Eq. (7) over $z$ :

$$
\begin{aligned}
E_{r \cdot s c}\left(z_{0}, R\right)= & \int_{-L / 2}^{L / 2} d E_{r} \\
= & \frac{Q}{4 \pi \epsilon_{0} L R}\left(\frac{z_{0}-L / 2}{\sqrt{\left(z_{0}-L / 2\right)^{2}+\frac{R^{2}}{\gamma^{2}}}}\right. \\
& \left.-\frac{z_{0}+L / 2}{\sqrt{\left(z_{0}+L / 2\right)^{2}+\frac{R^{2}}{\gamma^{2}}}}\right) .
\end{aligned}
$$

Substituting $E_{r \cdot s c}\left(z_{0}, R\right)$ for $E_{r \cdot s c}$ in Eq. (3), the transverse beam envelope equation for a pulsed beam is derived.

For example, in the case of $z_{0}=0$,

$$
E_{r \cdot s c}(0, R)=\frac{-Q}{4 \pi \epsilon_{0} R} \frac{1}{\sqrt{\frac{L^{2}}{4}+\frac{R^{2}}{\gamma^{2}}}} .
$$

Thus, the transverse beam envelope equation at the bunch center is derived as follows:

$$
\begin{aligned}
\frac{d^{2} R}{d t^{2}}= & \frac{e Q}{4 \pi \epsilon_{0} m_{0} \gamma^{3} R} \frac{1}{\sqrt{\frac{L^{2}}{4}+\frac{R^{2}}{\gamma^{2}}}}-\frac{e}{\gamma m_{0}} \\
& \times\left(E_{r}-\beta c B_{\theta}-\frac{\beta}{c} \cdot \frac{d R}{d t} E_{z}\right) .
\end{aligned}
$$

If $\gamma L \gg 2 R$, we can neglect $\frac{R^{2}}{\gamma^{2}}$. Then the space charge term given by Eq. (10) coincides with that of the envelope equation for a continuous beam given by Eq. (5), since $I=\frac{Q}{L} \beta c$.

\section{Longitudinal envelope equations}

To derive longitudinal beam envelope equations, we assume a uniformly charged cylindrical beam instead of a pencil beam, which propagates along the $z$ axis with an energy of $\gamma m_{0} c^{2}$, a bunch length of $L$, a radius of $R$, and a charge density of $\rho$, as shown in Fig. 3. The planes at both

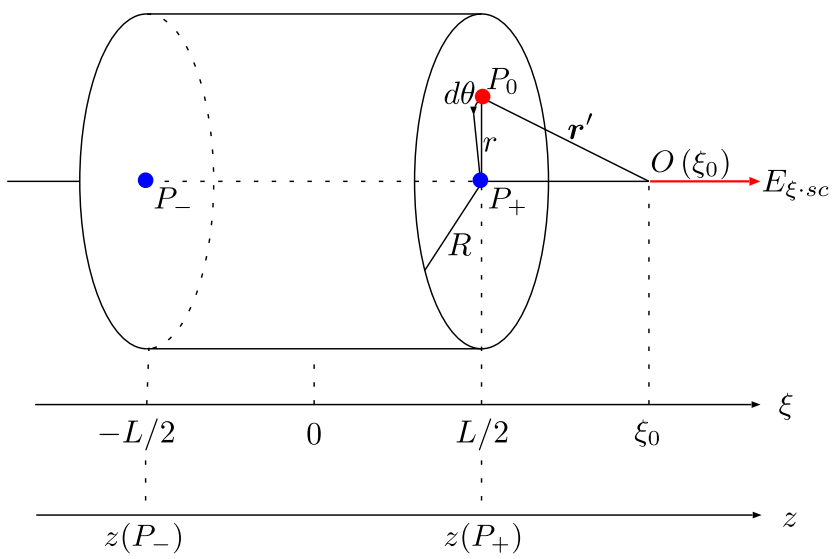

FIG. 3. Calculation model of space charge field from a cylindrical beam.

ends of the bunch are not warped in this cylindrical beam model. That is, the longitudinal velocities of all electrons located on a plane perpendicular to the $z$ axis are assumed to be equal. We also introduce another coordinate system $\xi$, which moves with the beam. The envelope equations are derived by tracing electrons, $P_{+}$and $P_{-}$, located at both ends of the bunch on the beam axis, in the $z$-coordinate system.

We start from the equation of motion, Eq. (2), in the same manner as that used to derive the transverse equation. Setting $v_{r}=v_{\theta}=0$, the $z$ component of Eq. (2) is

$$
\frac{d^{2} z}{d t^{2}}=-\frac{e}{\gamma^{3} m_{0}}\left(E_{\xi \cdot s c}+E_{z}\right)
$$

where $E_{\xi \cdot s c}$ is the space charge field and $E_{z}$ is an another external electric field. Using Eq. (6), $d E_{\xi \cdot s c}$ at point $O\left(\xi_{0}\right)$ in Fig. 3, which is produced by charge within a volume of $r d \theta d r d \xi$ at point $P_{0}$, is expressed as

$d E_{\xi \cdot s c}\left(\xi_{0}\right)=\frac{1}{4 \pi \epsilon_{0} \gamma^{2}} \frac{-\rho\left(\xi_{0}-\xi\right)}{\left[\left(\xi_{0}-\xi\right)^{2}+\frac{r^{2}}{\gamma^{2}}\right]^{3 / 2}} r d \theta d r d \xi$

$E_{\xi \cdot s c}$ can be derived by integrating Eq. (12) over $\theta, r$, and $\xi$. Since the total bunch charge $Q$ is $\pi R^{2} L \rho, E_{\xi \cdot s c}$ is

$$
\begin{aligned}
E_{\xi \cdot s c}\left(\xi_{0}\right)= & \int_{-L / 2}^{L / 2} \int_{0}^{R} \int_{0}^{2 \pi} d E_{\xi \cdot s c}\left(\xi_{0}\right) \\
= & \frac{Q}{2 \pi \epsilon_{0} R^{2} L}\left[-\sqrt{\left(\xi_{0}-\frac{L}{2}\right)^{2}+\frac{R^{2}}{\gamma^{2}}}\right. \\
& \left.+\sqrt{\left(\xi_{0}+\frac{L}{2}\right)^{2}+\frac{R^{2}}{\gamma^{2}}}+\left|\xi_{0}-\frac{L}{2}\right|-\left|\xi_{0}+\frac{L}{2}\right|\right] .
\end{aligned}
$$


Thus, $E_{\xi \cdot s c}$ at the position of each electron is

$$
\begin{aligned}
& E_{\xi \cdot s c}\left(P_{+}\right)=\frac{-Q}{2 \pi \epsilon_{0} R^{2} L}\left(L+\frac{R}{\gamma}-\sqrt{L^{2}+\frac{R^{2}}{\gamma^{2}}}\right) \\
& E_{\xi \cdot s c}\left(P_{-}\right)=-E_{\xi \cdot s c}\left(P_{+}\right) .
\end{aligned}
$$

The longitudinal beam envelope equations for electrons $P_{+}$and $P_{-}$are obtained by substituting Eqs. (14) into Eq. (11).

\section{Simultaneous beam envelope equations}

Combining Eqs. (10), (11), and (14), transverse and longitudinal simultaneous beam envelope equations are derived as ordinary differential equations. Replacing $L$ with $z\left(P_{+}\right)-z\left(P_{-}\right)$, the simultaneous beam envelope equations are derived as follows:

$$
\begin{aligned}
\frac{d^{2} R}{d t^{2}}= & -\frac{e}{\gamma^{3} m_{0}} E_{r \cdot s c}\left[R, z\left(P_{+}\right), z\left(P_{-}\right)\right] \\
& -\frac{e}{\gamma m_{0}}\left(E_{r}-\beta c B_{\theta}-\frac{\beta}{c} \cdot \frac{d R}{d t} E_{z}\right) \\
\frac{d^{2} z\left(P_{+}\right)}{d t^{2}}= & -\frac{e}{\gamma^{3} m_{0}} E_{\xi \cdot s c}\left[R, z\left(P_{+}\right), z\left(P_{-}\right)\right]-\frac{e}{\gamma^{3} m_{0}} E_{z} \\
\frac{d^{2} z\left(P_{-}\right)}{d t^{2}}= & \frac{e}{\gamma^{3} m_{0}} E_{\xi \cdot s c}\left[R, z\left(P_{+}\right), z\left(P_{-}\right)\right]-\frac{e}{\gamma^{3} m_{0}} E_{z},
\end{aligned}
$$

where

$$
\begin{aligned}
E_{r \cdot s c}\left[R, z\left(P_{+}\right), z\left(P_{-}\right)\right]= & -\frac{Q}{4 \pi \epsilon_{0} R} \frac{1}{\sqrt{\frac{\left[z\left(P_{+}\right)-z\left(P_{-}\right)\right]^{2}}{4}+\frac{R^{2}}{\gamma^{2}}}} \\
E_{\xi \cdot s c}\left[R, z\left(P_{+}\right), z\left(P_{-}\right)\right]= & -\frac{Q}{2 \pi \epsilon_{0} R^{2}\left[z\left(P_{+}\right)-z\left(P_{-}\right)\right]} \\
& \times\left(z\left(P_{+}\right)-z\left(P_{-}\right)+\frac{R}{\gamma}\right. \\
& \left.-\sqrt{\left[z\left(P_{+}\right)-z\left(P_{-}\right)\right]^{2}+\frac{R^{2}}{\gamma^{2}}}\right) .
\end{aligned}
$$

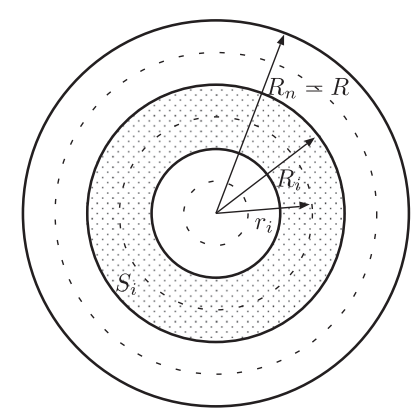

FIG. 4. Radial cross section of the beam used in the model for emittance calculation.

\section{E. Scheme of transverse emittance calculation}

In principle, transverse emittance can be calculated from the beam envelope equations.

As shown in Fig. 4, the beam is divided transversely into $n$ parts. The area between distances of $R_{i-1}$ and $R_{i}$ $\left(i=1, \ldots, n\right.$ and $\left.R_{n}=R\right)$ is denoted as $S_{i}$, and $r_{i}=$ $\left(R_{i-1}+R_{i}\right) / 2$. We trace electrons at distances of $r_{i}(i=1, \ldots, n)$ and $R$ from the center of the beam to evaluate the transverse charge distributions.

To trace all the electrons located at a distance of $r_{i}$, we have to solve simultaneous equations containing $n+3$ dependent variables, which consist of the three equations in Eq. (15) and the transverse equations for each $r_{i}(i=1, \ldots, n)$. The charge used in the transverse equations for $r_{i}$ should not be $Q$ because $r_{i}$ is smaller than $R$. Therefore, a correction function for $Q$, which is a function of $r_{i}$ and $R$, is required, which will be discussed in Sec. III B.

When the trace of each electron is solved, $\left\langle r^{2}\right\rangle,\left\langle r^{\prime 2}\right\rangle$, and $\left\langle r \cdot r^{\prime}\right\rangle^{2}$ can be obtained as weighted mean values:

$$
\langle A\rangle=\sum_{i=1}^{n} W_{i} \cdot A_{i},
$$

where each weight is $W_{i}=S_{i} /\left(\pi R^{2}\right)=(2 i-1) / n^{2}$. Then the emittance, $\epsilon_{x} \equiv 1 / 2 \sqrt{\left\langle r^{2}\right\rangle\left\langle r^{\prime 2}\right\rangle-\left\langle r \cdot r^{\prime}\right\rangle^{2}}$, can be calculated.

\section{MULTIPLE BEAM ENVELOPE EQUATIONS FOR ANALYSIS OF BEAM DYNAMICS}

\section{A. Scheme of multiple beam envelope equations}

Using the beam envelope equations discussed in Sec. II, only the radial beam size at the center of the bunch can be calculated. Therefore, the emittance derived by the method discussed in Sec. IIE is a slice emittance at the center of the bunch. To calculate the charge distribution of an entire bunch and the projected emittance, the bunch should be divided longitudinally into several slices.

A bunch segmentation model divided into $m$ bunches is shown in Fig. 5. Each traced electron is located not at the longitudinal center of each slice but at the segmentation boundary between each pair of slices. The electron located on the circumference of each segmentation boundary, $R_{j}$ $(j=1, \ldots, m+1)$, is used for the transverse trace, and the electron at $z_{j}$ on the $z$ axis is used for the longitudinal trace. The electron located at $r_{i j}$, which corresponds to $r_{i}$ in Fig. 4, is also used for the transverse trace. Note that $R_{j}$ differs from $R_{i}$ in Fig. 4.

$\beta_{j}$ is newly introduced as the normalized longitudinal velocity for each electron having suffix $j$ and is dominated by the following differential equation:

$$
\frac{d \beta_{j}}{d t}=\frac{1}{c} \frac{d^{2} z_{j}}{d t^{2}} .
$$




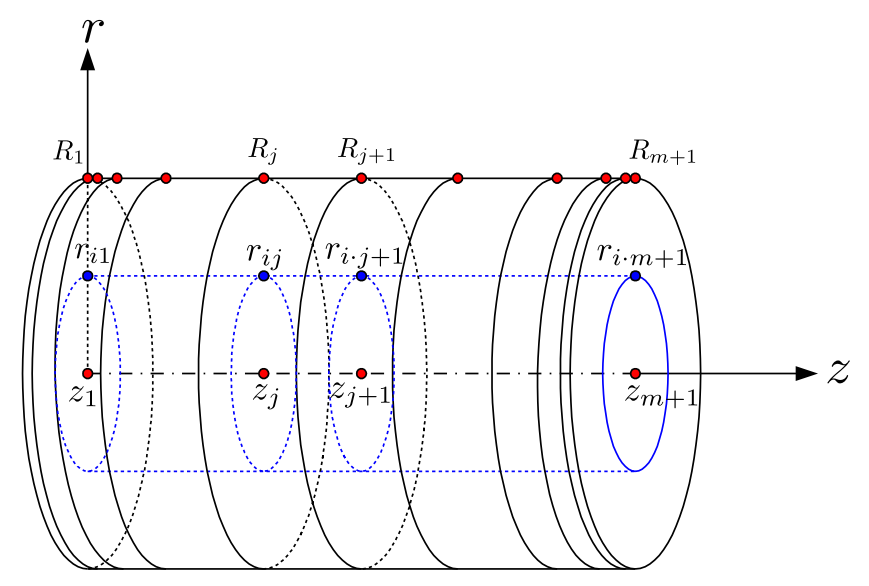

FIG. 5. Bunch segmentation model used to derive the multiple beam envelope equations. This indicates an initial bunch configuration.

The multiple beam envelope equations finally become simultaneous differential equations containing $(n+3)$ $(m+1)$ dependent variables, $R_{j}, r_{i j}, z_{j}$, and $\beta_{j}(i=$ $1, \ldots, n$ and $j=1, \ldots, m+1)$. The projected emittance of the bunch can be obtained by calculating weighted mean values in both the transverse and the longitudinal directions.

In this segmentation model, we assume that the longitudinal position and normalized longitudinal velocity of each electron located on a certain segmentation boundary $j$ are equal during the trace. That is, each slice is separated by planes perpendicular to the $z$ axis and is not be warped during the trace.

\section{B. Transverse space charge field calculations}

The transverse space charge fields are calculated on the basis of the pencil beam analysis in Sec. II, although these calculations are valid only when $\gamma L \gg 2 R$. To analyze a low-energy pulsed beam, more accurate models are required. If we adopt field analysis based on a cylindrical beam instead of a pencil beam, the differential equations become complicated. Therefore, we introduce a correction function for transverse space charge fields.

Here, we consider a pencil beam and a cylindrical beam with a length of $L$, where the radius of the cylindrical beam is $R_{c}$ and both beams have an energy of $\gamma m_{0} c^{2}$. Figure 6 shows the variations of $E_{r}$ along a line parallel to the beam axis at a distance of $r$. The center of the beam is located at $z=0$. The pencil beam field is calculated by Eq. (8), and the cylindrical beam field is calculated by integrating Eq. (8) numerically. The difference between these two patterns of field variation can be approximately expressed using two factors, a field correction factor, $E_{r}(0)_{\text {cylinder }} / E_{r}(0)_{\text {pencil }}$, and the intersection of the two fields as shown in Fig. 6. If these two correction factors are given, the cylindrical beam field between the points of intersection can be obtained from the pencil beam field by linear interpolation. These two correc- tion factors are functions of only two variables, $r / R_{c}$ and $2 r / \gamma L$, for the following reasons.

The cylindrical beam field is derived by integrating the pencil beam field given by Eq. (8) in the area within a radius of $R_{c}$. Therefore, the strength ratio of the cylindrical beam field to the pencil beam field is uniquely determined by the relative values of $r$ and the beam radius $R_{c}$. That is, the two correction factors are clearly functions of $r / R_{c}$.

Dividing the numerator and denominator of the righthand side of Eq. (8) by $L$, the variation of the pencil beam field with $z / L$ becomes a function of $r / \gamma L$. Therefore, the two correction factors are also functions of $2 r / \gamma L$.

Since the multiple beam envelope equations are complicated, it is necessary to solve them numerically. By preparing two-dimensional mapping data of the two correction factors as data files and loading them, the multiple equations can be computed with corrected $E_{r}$ as follows:

$$
E_{r \cdot \text { cylinder }}=F_{c}\left(\frac{r}{R_{c}}, \frac{2 r}{\gamma L}, \frac{z}{L}\right) \cdot E_{r \cdot \text { pencil }} \text {, }
$$

where $F_{c}$ is the correction function of the transverse space charge fields.

Note that $E_{r}$ outside the points of intersection in Fig. 6 is not corrected in the multiple envelope equations because the difference between $E_{r}$ for the pencil and cylindrical beams is small and its effect on the calculated trace is small.

Using this technique, the space charge field at a distance of $r_{i j}$ and inside the beam, as mentioned in Sec. IIE, can also be calculated.

External fields, such as fields in rf cavities or magnets, which can be expressed as functions of $z$ and $r$, can also be

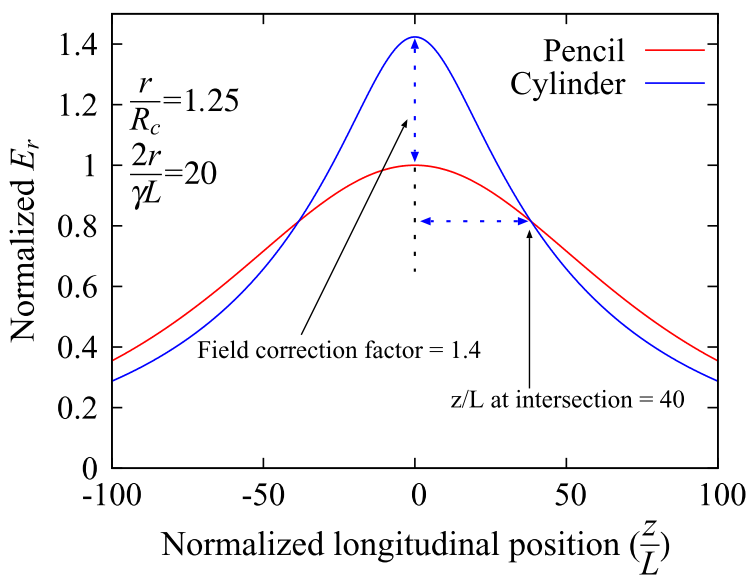

FIG. 6. Longitudinal variation of $E_{r}$ produced by a pencil beam and a cylindrical beam with a length of $L$. If two variables, $r / R_{c}=1.25$ and $2 r / \gamma L=20$ for example, are given, the two correction factors, the field correction factor and $z / L$ at the point of intersection, are uniquely determined. The value of $E_{r}$ for a cylindrical beam can be obtained from the pencil beam field and the two correction factors. 
included in the multiple envelope equations in the same manner as that used to correct $E_{r}$.

\section{Summations of space charge fields}

The space charge fields in the multiple envelope equations are expressed as sums of the fields produced from each segmentation slice $k$. From Eqs. (8) and (13), the space charge fields, $E_{r_{i j} s c}, E_{R_{j} s c}$, and $E_{\xi_{j} s c}$, acting on a certain electron being traced are

$$
\begin{aligned}
E_{r_{i j} s c}= & \frac{1}{4 \pi \epsilon_{0}} \sum_{k=1}^{m} \frac{F_{c}\left(\frac{r_{i j}}{R(k)}, \frac{2 r_{i j}}{\gamma(k) L(k)}, \frac{z_{j}-z(k)}{L(k)}\right) \cdot Q(k)}{r_{i j} L(k)} \\
& \times\left(\frac{z_{j}-z_{k+1}}{\sqrt{\left(z_{j}-z_{k+1}\right)^{2}+\frac{r_{i j}^{2}}{\gamma(k)^{2}}}}-\frac{z_{j}-z_{k}}{\sqrt{\left(z_{j}-z_{k}\right)^{2}+\frac{r_{i j}^{2}}{\gamma(k)^{2}}}}\right)
\end{aligned}
$$

$$
\begin{aligned}
E_{R_{j} s c}= & \frac{1}{4 \pi \epsilon_{0}} \sum_{k=1}^{m} \frac{F_{c}\left(\frac{R_{j}}{R(k)}, \frac{2 R_{j}}{\gamma(k) L(k)}, \frac{z_{j}-z(k)}{L(k)}\right) \cdot Q(k)}{R_{j} L(k)} \\
& \times\left(\frac{z_{j}-z_{k+1}}{\sqrt{\left(z_{j}-z_{k+1}\right)^{2}+\frac{R_{j}^{2}}{\gamma(k)^{2}}}}-\frac{z_{j}-z_{k}}{\sqrt{\left(z_{j}-z_{k}\right)^{2}+\frac{R_{j}^{2}}{\gamma(k)^{2}}}}\right)
\end{aligned}
$$

$$
\begin{aligned}
E_{\xi_{j} s c}= & \frac{1}{2 \pi \epsilon_{0}} \sum_{k=1}^{m} \frac{Q(k)}{R(k)^{2} L(k)}\left(-\sqrt{\left(z_{j}-z_{k+1}\right)^{2}+\frac{R(k)^{2}}{\gamma(k)^{2}}}\right. \\
& \left.+\sqrt{\left(z_{j}-z_{k}\right)^{2}+\frac{R(k)^{2}}{\gamma(k)^{2}}}+\left|z_{j}-z_{k+1}\right|-\left|z_{j}-z_{k}\right|\right),
\end{aligned}
$$

where $k$ is the number of slices. $L(k), Q(k), R(k), z(k)$, and $\gamma(k)$ are parameters of $k$, that is,

$$
\begin{aligned}
L(k) & \equiv z_{k+1}-z_{k} \\
Q(k) & \equiv \Delta t_{k} / T \cdot Q=\mathrm{const} \\
R(k) & \equiv\left(R_{k+1}+R_{k}\right) / 2 \\
z(k) & \equiv\left(z_{k+1}+z_{k}\right) / 2 \\
\gamma(k) & \equiv\left(\gamma_{k+1}+\gamma_{k}\right) / 2,
\end{aligned}
$$

$T$ is the length of time for the entire bunch, and $\Delta t_{k}$ is the length of time for slice $k$.
Equations (20)-(22) are substituted into the envelope equations, Eqs. (15), in place of Eqs. (16).

\section{Beam emission from a cathode and image charge effects}

To calculate the beam emission from a cathode, we assume that the cathode surface is at $z=0$. Initially, the positions of all electrons are set on the cathode surface. The electron with suffix $j=m+1$, as shown in Fig. 5, at the head of the bunch is emitted from the cathode at $t=$ $t_{m+1}=0$, and the other electron with suffix $j \neq m+1$ is emitted at $t=t_{j}=\sum_{k=j}^{m} \Delta t_{k}$. In practice, when solving the differential equations, $\frac{d R_{j}}{d t}, \frac{d r_{i j}}{d t}$, and $\frac{d z_{j}}{d t}$ are zero when $t<t_{j}$.

We consider the condition that electron $j+1$ is emitted and electron $j$ is not emitted, that is, $t_{j+1}<t<t_{j}$. In this case, $Q(k)$ in Eqs. (20)-(22) should be set as follows:

$$
Q(k) \equiv \begin{cases}\Delta t_{k} / T \cdot Q & (k \geq j+1) \\ \left(t-t_{j+1}\right) / T \cdot Q & (k=j) \\ 0 & (k \leq j-1) .\end{cases}
$$

Then the emission from the cathode can be calculated continuously.

The image charge effects at the cathode can be simply calculated by placing an image electron with the positive charge at a symmetrical point about the cathode, which is assumed to be a perfect conductor. The image electron moves in the opposite direction to the real electron. When the suffix of the image slice is $-k, Q(-k)$ should be defined as follows:

$$
Q(-k) \equiv \begin{cases}-\Delta t_{k} / T \cdot Q & (k \geq j+1) \\ -\left(t-t_{j+1}\right) / T \cdot Q & (k=j) \\ 0 & (k \leq j-1) .\end{cases}
$$

Note that summations in Eqs. (20)-(22) have to be performed from $k=-m$ to $m(k \neq 0)$.

\section{BEAM DYNAMICS CALCULATIONS USING THE MULTIPLE BEAM ENVELOPE EQUATIONS}

The multiple beam envelope equations are numerically solved using Octave [14]. We use the "lsode" command with the non-stiff option in Octave to analyze the differential equations. The multiple envelope equations have many dependent variables and are complicated. Therefore, to solve the simultaneous differential equations containing $(n+3)(m+1)$ variables, the equations are separated into $n$ sets of equations containing $4(m+1)$ variables, which are $R_{j}, r_{i j}, \beta_{j}$, and $z_{j}(j=1, \ldots, m+1)$. Because there are no relations among each $r_{i j}$ with respect to $i$. The calculations have to be performed $n$ times, corresponding to $i=1, \ldots, n$. 


\section{A. Traces in free space}

As a basic model, the trace results in free space are discussed here. Initially, a bunch with a longitudinal length of $3 \mathrm{~mm}$, a beam radius of $0.5 \mathrm{~mm}$, a charge of $1 \mathrm{nC}$ per bunch, an energy of $1 \mathrm{MeV}$, and an emittance of $0 \mathrm{mrad}$ is set at $z=0$. Both the transverse and the longitudinal charge densities are assumed to be uniform. The bunch is traced for a distance of $0.1 \mathrm{~m}$ along the $z$ axis in free space. The manner of bunch segmentation is the same as that shown in Fig. 5, where $m=10, n=10, z_{1}=0$, and $z_{m+1}=3 \mathrm{~mm}$. The length of the segmentation slices at both ends of the bunch is set to be shorter than that of the middle slices as shown in Fig. 5.

The calculation results, along with those for a particletracking simulation code [13] developed by the authors, are shown in Fig. 7. The horizontal axis is expressed not as a time scale but as a distance scale, where $z(t)=$ $\int_{0}^{t} \beta(t) c \cdot d t$. In the simulation code, initial particles are aligned using Hammersley's sequence [15] to reduce the random noise caused by the distributions of particle
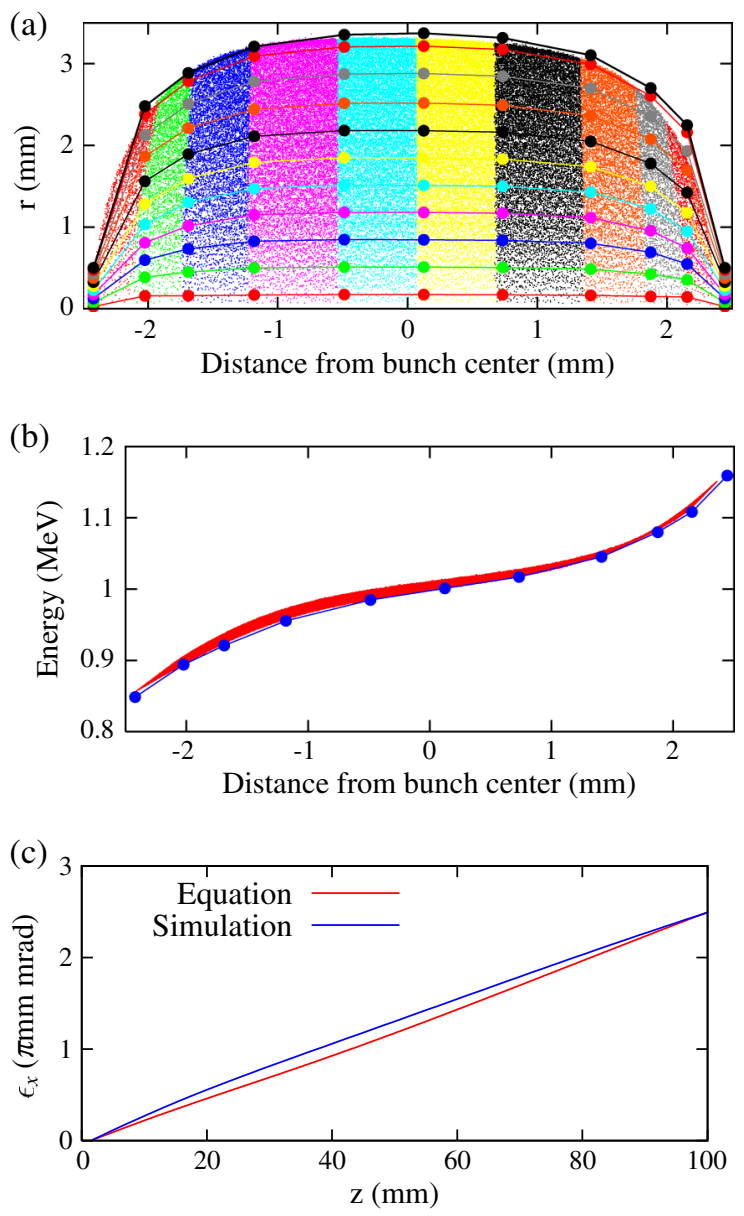

FIG. 7. Results of free-space traces with an energy of $1 \mathrm{MeV}$ and a charge per bunch of $1.0 \mathrm{nC}$. (a) Bunch shapes after $0.1 \mathrm{~m}$ trace. (b) Energy distributions in the bunch after $0.1 \mathrm{~m}$ trace. (c) Time evolutions of emittances. positions. The number of particles used in the simulation code is $1 \times 10^{5}$.

Figure 7(a) shows bunch shapes after tracing for a distance of $0.1 \mathrm{~m}$. Each dot on the solid lines is an electron traced using the envelope equations. The clouds of small dots are the particles in the simulation code. The particles are color coded according to the initial segmentation slices used in the multiple envelope equations.

Although the simulation code is three dimensional, in Fig. 7(a) $r$ is plotted as a variable on the vertical axis to compare it with the two-dimensional envelope equations.

Figure 7(b) shows the energy distributions in the bunch after tracing for a distance of $0.1 \mathrm{~m}$. The dots on the line represent the results obtained by the beam envelope equations.

Figure 7(c) shows the time evolutions of the rms normalized emittances, which are defined as

$$
\begin{aligned}
& \epsilon_{x} \equiv\langle\gamma\rangle\langle\beta\rangle \sqrt{\left\langle x^{2}\right\rangle\left\langle x^{\prime 2}\right\rangle-\left\langle x \cdot x^{\prime}\right\rangle^{2}} \quad \text { (simulation) } \\
& \epsilon_{x} \equiv \frac{1}{2}\langle\gamma\rangle\langle\beta\rangle \sqrt{\left\langle r^{2}\right\rangle\left\langle r^{\prime 2}\right\rangle-\left\langle r \cdot r^{\prime}\right\rangle^{2}} \quad \text { (equation) }
\end{aligned}
$$

Even when $2 R / \gamma L$ is 0.46 in Fig. 7(a), for which the treatment of a short bunch with low energy is required, the results obtained by the envelope equations and the simulation code show good agreement.

\section{B. Emittance accuracy}

Figure 8 shows the results of free-space traces with an energy of $6 \mathrm{MeV}$. The bunch shapes are in good agreement with each other, although the emittances obtained by the envelope equations are less than those obtained by the simulation. Figure 9 shows the emittance dependence on the number of particles in the simulation at $z=0.1$. The emittances are normalized by their values when $n=$ $1 \times 10^{5}$. Since the beam size remains small, and thus the charge density is large, for an energy of $6 \mathrm{MeV}$, the effect of the random noise of the particle distribution is enhanced. Therefore, the variation of emittance with respect to the number of particles is larger than that for an energy of $1 \mathrm{MeV}$, and the emittance does not converge even when $n=2 \times 10^{5}$.

The number of particles does not appear explicitly in the envelope equations, and the emittance obtained by the simulation code is expected to become close to that obtained by the equations with increasing the number of particles. Therefore, the emittance calculated by the equations is more accurate than that calculated by the simulation code.

In addition, calculation time of the envelope equations is much shorter than that of the simulation. Though depending on a type of CPU, it is an order of a week for the simulation when $n=2 \times 10^{5}$ using a single core of Xeon W5590 3.33 GHz. In contrast, it is an order of a minute for the envelope equations. 

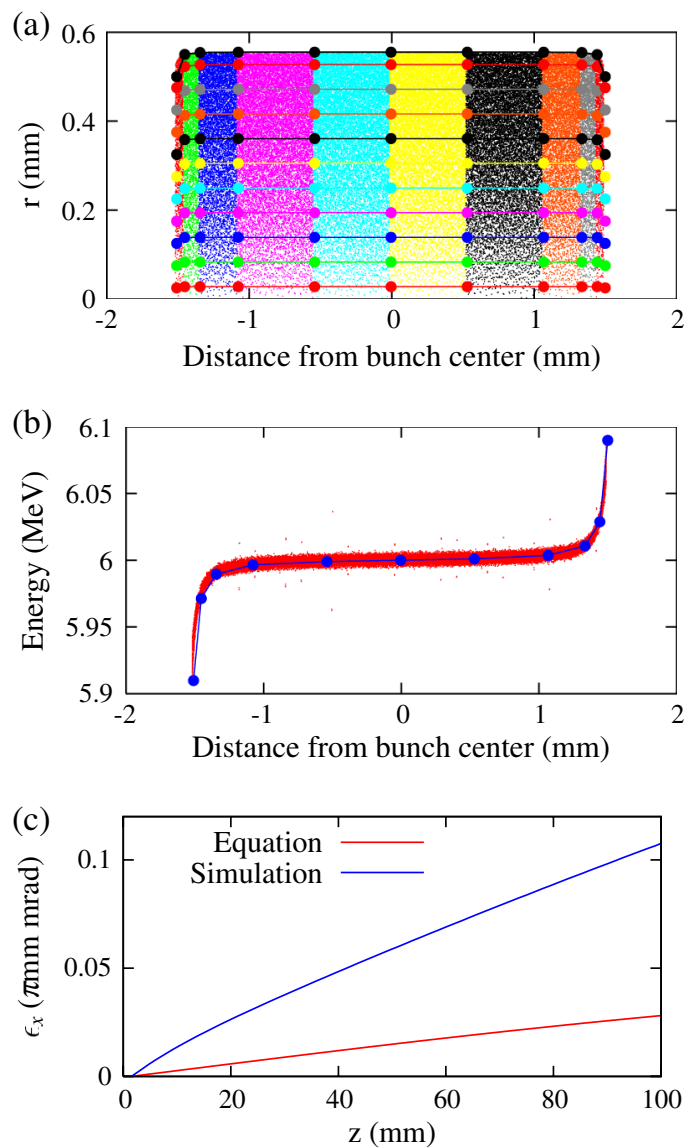

FIG. 8. Results of free-space traces with an energy of $6 \mathrm{MeV}$ and a charge per bunch of $1.0 \mathrm{nC}$. (a) Bunch shapes after $0.1 \mathrm{~m}$ trace. (b) Energy distributions in the bunch after $0.1 \mathrm{~m}$ trace. (c) Time evolutions of emittances.

\section{Beam dynamics in BNL-type rf gun cavity}

The beam dynamics in the 1.6 cell BNL-type rf gun cavity is calculated. A list of the initial parameters is shown in Table I. The laser spot size and energy at the exit of the cavity are obtained from Ref. [16]. The initial thermal emittance is not taken into account, thus it is assumed to be $0 \mathrm{mrad}$. The calculations include only emission from the

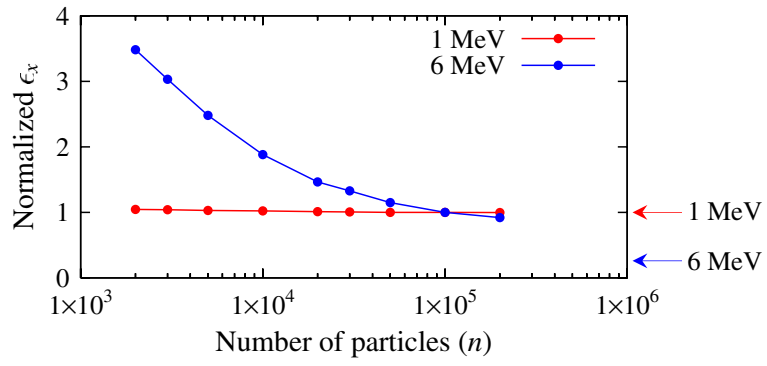

FIG. 9. Emittance dependence on number of particles in the particle simulation code. The emittances are normalized to those when $n=1 \times 10^{5}$. The emittances calculated by the envelope equations are also shown as arrows on the right-hand side.
TABLE I. Parameters for beam dynamics calculations in BNL-type rf gun cavity.

\begin{tabular}{lc}
\hline \hline Laser length & $10 \mathrm{ps}$ uniform \\
Laser spot size & $\phi 1.3 \mathrm{~mm}$ uniform \\
Maximum electric field on the & $120.8 \mathrm{MV} / \mathrm{m}$ \\
cathode & $5.75 \mathrm{MeV}$ \\
Energy at the exit of the cavity & $\sin 25 \mathrm{deg}$. \\
$\begin{array}{l}\text { Initial rf phase (when the head of } \\
\text { the bunch is emitted) }\end{array}$ & $0 \mathrm{mrad}$ \\
\hline Initial emittance
\end{tabular}

cathode and acceleration in the cavity and do not include any other external fields such as focusing fields.

Figure 10 shows calculation results for a charge of $0.1 \mathrm{nC}$ per bunch including the effects of image charge fields at the cathode. The number of particles used in the simulations is $1 \times 10^{5}$. The bunch shapes and energy distributions at the exit of the cavity $(z=150 \mathrm{~mm})$ are shown.

Figure 10(c) shows time evolutions of the emittances. The emittances oscillate in the rf cavity. These oscillations are caused by each slice having a different time evolution
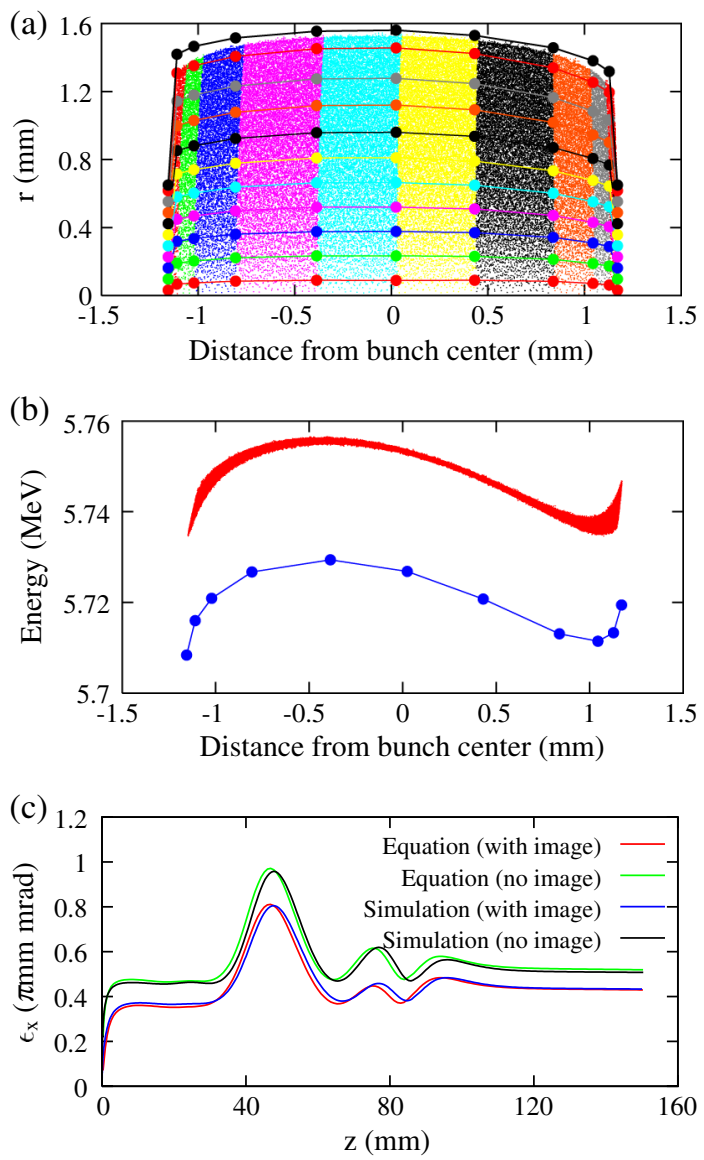

FIG. 10. Beam dynamics calculations for a charge of $0.1 \mathrm{nC}$ per bunch in the BNL-type rf gun cavity. (a) Bunch shapes after the rf gun cavity. (b) Energy distributions after the rf gun cavity. (c) Time evolutions of emittances. The cavity structure is located from $z=0$ to $77.67 \mathrm{~mm}$. 
of the rotation angle in the phase space. Since the envelope equations can calculate not only a slice emittance but the total projected emittance of the bunch, the results obtained by the equations agree with those of the simulation code.

Emittances without image charge fields at the cathode are also plotted in Fig. 10(c); the effects of the image charge fields obtained from the equations and the simulation also coincide with each other.

Figure 11 shows the emittance dependence on the number of particles. The emittances are normalized by their value when $n=1 \times 10^{5}$. Similarly to the results of the free-space traces, the emittance obtained by the simulation becomes closer to that obtained by the equations with increasing the number of particles.

Figure 12 shows the results for a charge of $1 \mathrm{nC}$ per bunch. The time evolution pattern of emittance obtained by the equations and the simulation coincide with each other, although the value of emittance is slightly different. There are two possible causes of this difference, one related to the simulation and one related to the envelope equations.

The cause related to the simulation is an error in the calculation of the image charge fields. In our tracking simulation code, since the position of the image particle $r$ is the same as that of the corresponding real particle, the distance between the two particles becomes short near the cathode and a very large interaction force appears. Thus, a shield length is introduced, and the interaction between the real and image particles is not calculated if the distance between them is less than this length. However, the shield length cannot be determined logically in the simulation. Since this treatment is not required in the analytical method, the results for the envelope equations are expected to be more accurate.

The cause related to the envelope equations is the warp of the slice shape. In the envelope equations, each slice must be separated by a plane perpendicular to the $z$ axis according to the assumption in the segmentation bunch model. However, the results of the simulation in Fig. 12(a) indicate that each segmentation boundary becomes curved, because $E_{\xi \cdot s c}$ on the $z$ axis and that on an off-axis line are different. This is one of the limitations of our multiple envelope equation analysis. Both of the above two problems are caused by a high charge density.

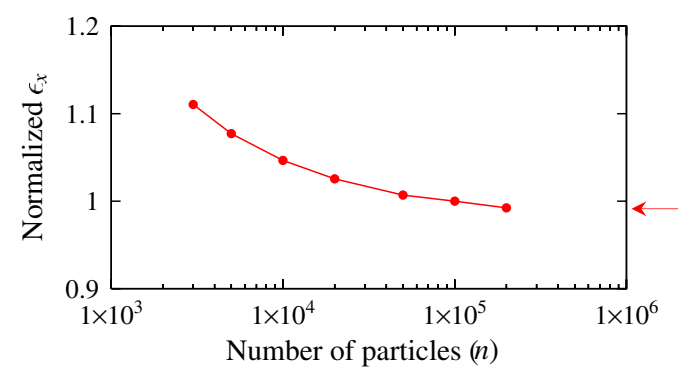

FIG. 11. Emittance dependence on number of particles in the particle simulation code. The emittance obtained by the envelope equations is shown as an arrow. The charge per bunch is $0.1 \mathrm{nC}$.
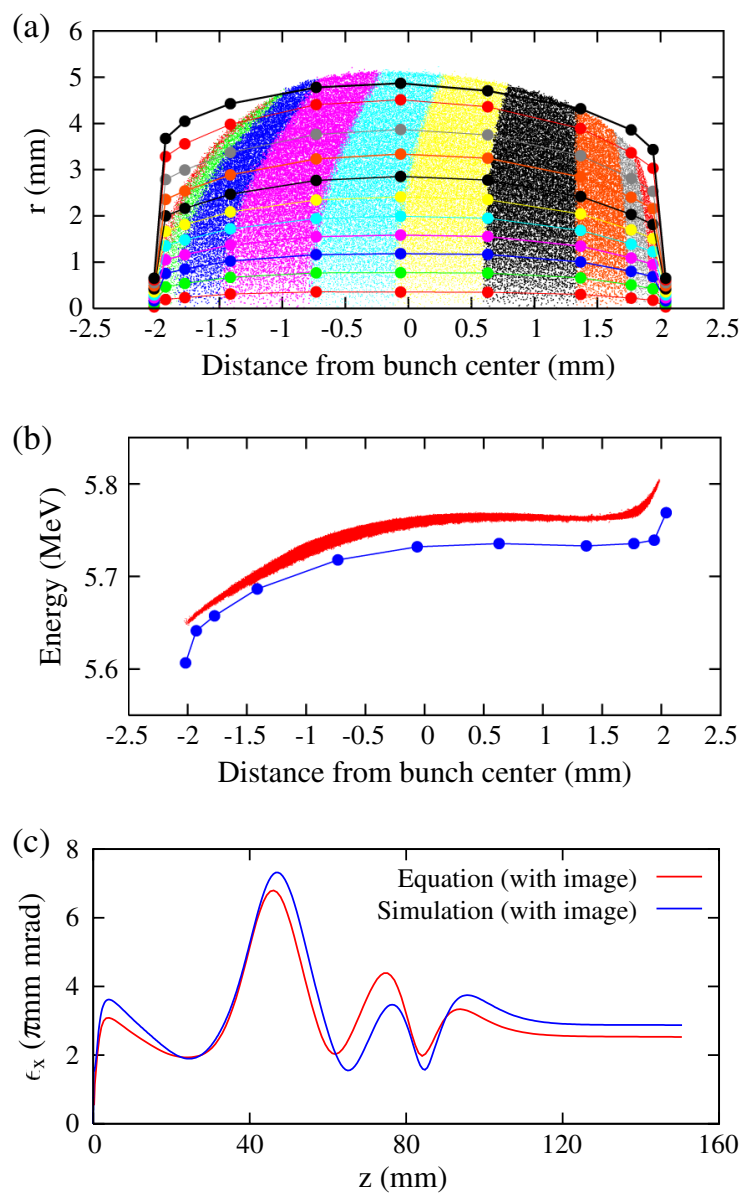

FIG. 12. Beam dynamics calculations for a charge of $1 \mathrm{nC}$ per bunch in the BNL-type rf gun cavity. (a) Bunch shapes after the rf gun cavity. (b) Energy distributions after the rf gun cavity. (c) Time evolutions of emittances.

Note that each solid line in a calculated bunch shape must not intercross. The crossing has possibility to occur when the charge density is high. Though insofar as each slice is not warped in the simulation, it is expected not to occur. This can be checked from a calculated result of the envelope equations, and there is no crossing in Fig. 12(a).

Figure 13 shows the calculated emittance as the function of charge per bunch evaluated at $z=0.15 \mathrm{~m}$. Since the calculations do not take into account the initial thermal emittance, the emittance is expressed as

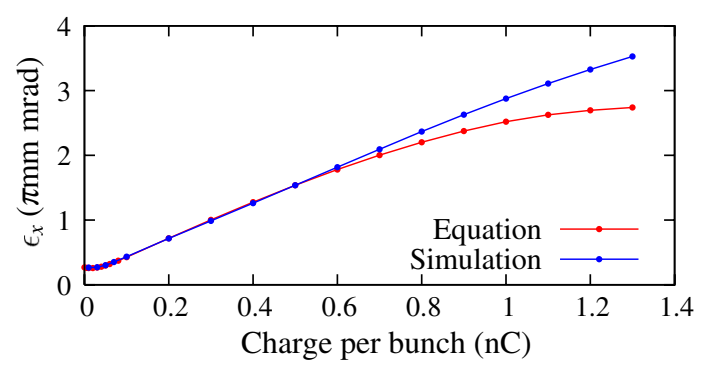

FIG. 13. Emittance dependence on charge per bunch. The number of particles in the simulation is $1 \times 10^{5}$. 
$\epsilon_{x}=\sqrt{\epsilon_{\mathrm{rf}}\left(\sigma_{r}\right)^{2}+\epsilon_{s c}(q)^{2}}$, where, $\epsilon_{\mathrm{rf}}$ is the rf emittance, $\epsilon_{s c}$ is the space charge emittance, $\sigma_{r}$ is the rms bunch radius, and $q$ is the charge per bunch. In this case, the rf emittance is $0.25 \pi \mathrm{mm}$ mrad from Fig. 13 because $\epsilon_{s c}(0)=0$. The applicable range of the multiple envelope equations under the conditions in Table $I$ is under $0.5 \mathrm{nC} /$ bunch, since the emittances obtained by the equations and the simulation coincide with each other in this range. This range is often used in recently built XFEL facilities $[17,18]$.

\section{CONCLUSION}

A new semianalytical method for investigating the beam dynamics of electron injectors was developed by deriving multiple beam envelope equations incorporating a bunch segmentation model and a transverse space charge field correction scheme.

The advantage of this method is its unique assumption for the beam parameters. We assume that each segmentation slice is not warped in the calculations. Although if the beam energy is low and the charge density is large, this condition is not satisfied, in practice this condition is usually satisfied.

We have performed beam dynamics calculations to obtain traces in free space and in the BNL-type rf gun cavity by comparing the analytical solutions with those obtained by the simulation. In most cases, including that of an rf gun cavity with a low charge, the bunch shape and the energy distribution in the bunch agree with those obtained by the simulation. The emittances obtained by the simulation become closer to those obtained analytically with increasing number of particles used in the simulation. Therefore, the analytically obtained emittances are expected to coincide with converged values obtained by the simulation.

On the other hand, significantly warped slices were found in the simulation result for the trace of a $1 \mathrm{nC}$ per bunch in the BNL-type rf gun cavity. The applicable range of the analytical solutions for the BNL-type rf gun was under $0.5 \mathrm{nC}$ per bunch, since each slice does not warp in this range. This range is often used in recently built XFEL facilities.
[1] Linac Coherent Light Source (LCLS) Conceptual Design Report No. SLAC-R-593, 2002.

[2] The European X-Ray Free-Electron Laser Technical Design Report No. 2006-097, 2006.

[3] SCSS X-FEL R\&D Group, SCSS X-FEL Conceptual Design Report, edited by T. Shintake and T. Tanaka (RIKEN Harima Institute/SPring-8, Sayo, Japan, 2005).

[4] J.D. Lawson, The Physics of Charged-Particle Beam (Oxford Science Publications, New York, 1988).

[5] K. J. Kim, Nucl. Instrum. Methods Phys. Res., Sect. A 275, 201 (1989).

[6] L. Serafini and J. B. Rosenzweig, Phys. Rev. E 55, 7565 (1997).

[7] C. K. Allen and M. Reiser, Phys. Rev. E 55, 7591 (1997).

[8] M. Ferrario, A. Mosnier, L. Serafini, F. Tazzioli, and J.-M. Tessier, Part. Accel. 52, 1 (1996).

[9] http://www.pulsar.nl/gpt.

[10] G. Pöplau and K. Flöttmann, in Proceedings of the Tenth European Particle Accelerator Conference, Edinburgh, Scotland (EPS-AG, Edinburgh, Scotland, 2006), p. 2203.

[11] L. M. Young and J.H. Billen, Los Alamos National Laboratory Report No. LA-UR-96-1835, 2004.

[12] J. Qiang, S. Lidia, R. D. Ryne, and C. Limborg-Deprey, Phys. Rev. ST Accel. Beams 9, 044204 (2006).

[13] A. Mizuno, T. Asaka, H. Dewa, T. Kobayashi, S. Suzuki, T. Taniuchi, H. Tomizawa, K. Yanagida, H. Hanaki, and M. Uesaka, Nucl. Instrum. Methods Phys. Res., Sect. A 528, 387 (2004).

[14] http://www.octave.org.

[15] J. Gonichon, S. C. Chen, L. C-L. Lin, and R. J. Temkin, in Proceedings of the 1993 Particle Accelerator Conference, Washington, DC (IEEE, New York, 1993), p. 2696.

[16] R. Akre et al., Phys. Rev. ST Accel. Beams 11, 030703 (2008).

[17] A. Brachmann, F. J. Decker, P. Emma, R. Iverson, P. Stefan, J. Turner, and F. Zhou, in Proceedings of IPAC2011, San Sebastián, Spain (EPS-AG, Spain, 2011), p. 3200.

[18] T. Shintake et al., Phys. Rev. ST Accel. Beams 12, 070701 (2009). 